PROCEEDINGS OF THE

AMERICAN MATHEMATICAL SOCIETY

Volume 132, Number 3, Pages 687-690

S 0002-9939(03)07178-8

Article electronically published on July 16, 2003

\title{
LOCAL INTERSECTIONS OF PLANE ALGEBRAIC CURVES
}

\author{
CLAIRE MOURA
}

(Communicated by Michael Stillman)

\begin{abstract}
We determine the maximum punctual order of contact between two plane algebraic curves, of which one is reduced. We prove that, generically on the reduced curve, this quantity is always strictly bounded by the product of the degrees of the curves.
\end{abstract}

\section{INTRODUCTION}

Two projective algebraic curves of $\mathbf{P}^{2}(\mathbf{C})$ that do not have any common component meet in a finite number of points. Bezout's theorem states that the total intersection multiplicity is given by the product of the degrees of the curves.

We focus on punctual intersection multiplicity of plane algebraic curves: let $\gamma$ be an affine algebraic curve in $\mathbf{C}^{2}$, irreducible, defined by a reduced polynomial of degree $p$. Given an integer $q$, we study the order of contact, at a fixed point $P$ of $\gamma$, between $\gamma$ and the ideal generated by any polynomial $Q$ of degree inferior or equal to $q$, whose zero locus does not contain $\gamma$. This quantity will be denoted by $[Q, \gamma]_{P}$. It is the order at $t=0$ of $Q$ composed with a Puiseux parametrization $\psi(t)$ of the curve $\gamma$ at $P$. By Bezout's theorem, the set $\left\{[Q, \gamma]_{P}: \operatorname{deg} Q \leq q\right\}$ is finite. Its maximum $M(\gamma, q, P)$ is also known as the Bautin index of the curve $\gamma$ at the point $P$, with respect to degree $q$. It has been studied and bounded in the more general setting of an analytic curve $\gamma$, solution of a polynomial vector field (see, for example, Gabrielov 11, Yomdin 44). In the case when $\gamma$ is plane algebraic, we obtain the following estimates for the Bautin index that only depend on the involved degrees.

Theorem 1. For a generic point $P$ on $\gamma$ :

If $q<p$, then:

$$
M(\gamma, q, P)=\max \left\{[Q, \gamma]_{P}: \operatorname{deg} Q \leq q\right\}=\left(\begin{array}{c}
q+2 \\
2
\end{array}\right)-1=\frac{q^{2}+3 q}{2}<p q .
$$

If $q \geq p$, then:

$$
M(\gamma, q, P)=\left(\begin{array}{c}
q+2 \\
2
\end{array}\right)-\left(\begin{array}{c}
q-p+2 \\
2
\end{array}\right)-1=p q-\frac{p^{2}-3 p+2}{2} .
$$

Received by the editors January 16, 2001 and, in revised form, November 5, 2002.

2000 Mathematics Subject Classification. Primary 14H50.

This work was partially supported by CNRS and EU contract ERBFMRXCT 960040 Valladolid (Spain). 
In particular, this proves that, if we fix a generic point of $\gamma$, the global intersection multiplicity $p q$ cannot be concentrated at this point, for any choice of polynomial $Q$.

To establish this result, we consider the space $E$ of polynomials of degree less than $q$, restricted to the curve $\gamma$. The maximum order of contact is interpreted as the index of stabilization of a decreasing sequence, starting at $E$, of vector subspaces of $E$. We prove that, generically on $\gamma$, this sequence is strictly decreasing, and stabilizes after $\operatorname{dim}_{\mathbf{C}} E-1$ steps. Theorem 1 follows. As a corollary of the proof, we derive the upper semi-continuity on the regular part of $\gamma$ of the function $P \mapsto M(\gamma, q, P)$.

\section{Proof of Theorem 1: The Wronskian argument}

We denote by $x$ and $y$ the canonical coordinates on $\mathbf{C}^{2}$, by $\mathbf{C}[x, y]_{q}$ the set of polynomials of degree less than $q$, and by $(F=0) \operatorname{deg} F=p$, the equation of $\gamma$. Let $E$ be the image of $\mathbf{C}[x, y]_{q}$ by the canonical surjection $s: \mathbf{C}[x, y] \rightarrow \frac{\mathbf{C}[x, y]}{(F)}$.

If $q<p$, then $E \simeq \mathbf{C}[x, y]_{q}$ since no nontrivial polynomial of $\mathbf{C}[x, y]_{q}$ belongs to the ideal generated by $F$. Then $\operatorname{dim}_{\mathbf{C}} E=\left(\begin{array}{c}q+2 \\ 2\end{array}\right)$.

If $q \geq p$, then $\operatorname{ker} s=\{F G, \operatorname{deg} G \leq q-p\}$ since $\gamma$ is irreducible and

$$
E \simeq \frac{\mathbf{C}[x, y]_{q}}{\mathbf{C}[x, y]_{q-p}}
$$

so that $\operatorname{dim}_{\mathbf{C}} E=\left(\begin{array}{c}q+2 \\ 2\end{array}\right)-\left(\begin{array}{c}q-p+2 \\ 2\end{array}\right)$.

From now on, we will set $l$ for $\operatorname{dim}_{\mathbf{C}} E$. We fix a point $P$ on $\gamma$ and define, for each integer $n$, the following vector subspace of $E$ :

$$
E_{n}(P)=\left\{Q \in E:[Q, \gamma]_{P} \geq n\right\} .
$$

Remark 1. Because of Bezout's theorem, the decreasing sequence $\left(E_{n}(P)\right)_{n \in \mathbf{N}}$ stabilizes on $\{0\}$. If $n_{0}$ is the first integer $n$ such that $E_{n}(P)=\{0\}$, then $M(\gamma, q, P)=$ $n_{0}-1$.

Following Miranda's terminology in [3], we introduce the notion of $q$-Gap.

Definition. We will say that $n \in \mathbf{N}$ is a $q$-Gap at $P$ if the space $E_{n}(P)$ is strictly contained in $E_{n-1}(P)$.

Proposition 1. Let $P$ be a point of $\gamma^{*}$, the regular part of $\gamma$. If $n$ is a $q$-Gap at $P$, then $\operatorname{dim}_{C} E_{n}(P)=\operatorname{dim}_{C} E_{n-1}(P)-1$.

Proof. We write a basis of $E$ in a neighbourhood of the point $P$, using germs of holomorphic functions on $\gamma$. Let $z$ be a uniformizing parameter for $\gamma$ at $P$ and let $\left\{g_{1}(z), \ldots, g_{l}(z)\right\}$ be a basis of $E$. A polynomial $Q \in E$ decomposes in a neighbourhood of $P$ as a combination since $c_{1} g_{1}+\ldots+c_{l} g_{l}, c_{i} \in \mathbf{C}, i=1, \ldots, l$. Hence, $Q$ belongs to $E_{n}(P)$ if and only if $c_{1} g_{1}+\ldots+c_{l} g_{l}$ has order greater than $n$ at $z=0$, that is, $\left(c_{1}, \ldots, c_{l}\right)$ satisfies the following linear system:

$$
\left\{\begin{array}{lll}
c_{1} g_{1}(0)+\ldots+c_{l} g_{l}(0) & = & 0, \\
c_{1} g_{1}^{\prime}(0)+\ldots+c_{l} g_{l}^{\prime}(0) & = & 0, \\
\vdots & & \\
c_{1} g_{1}^{(n-1)}(0)+\ldots+c_{l} g_{l}^{(n-1)}(0) & = & 0 .
\end{array}\right.
$$


Whence the Proposition.

Remark 2. It follows from Remark 1 and Proposition 1 that the number of $q$-Gaps at a regular point of $\gamma$ is exactly $l$, that is to say, the system (1) has rank $l$. Now, let $P \in \gamma^{*}$ and let $\left(1, e_{2}, \ldots, e_{l}\right)$ be the vector of $q$-Gaps at $P$. Of course, $e_{i}$ is greater than or equal to $i$, for all $i=2, \ldots, l$. Moreover, the definition of a $q$ Gap implies that $E_{e_{l}}(P)=\{0\}$ and $\operatorname{dim}_{\mathbf{C}} E_{e_{l-1}}(P)=\operatorname{dim}_{\mathbf{C}} E_{e_{l-1}+1}(P)=\ldots=$ $\operatorname{dim}_{\mathbf{C}} E_{e_{l}-1}(P)=1$, so that $M(\gamma, q, P)=e_{l}-1$.

Remark 3. In the case $q<p, l$ is exactly the number of monomials of degree less than $q$. The basis $\left\{g_{i}\right\}$ is simply obtained by restricting the $l$ monomials to the germ of $\gamma$ at $P$. Then Remark 2 means that there is a unique polynomial $Q$ (up to multiplication by a nonzero constant) that achieves the maximum order of contact $e_{l}-1$ with $\gamma$ at $P$. It is given by $c_{1}+c_{2} x+c_{3} y+\ldots+c_{l} y^{q},\left(c_{1}, \ldots, c_{l}\right)$ being a solution of the system (1), where the $g_{i}$ 's are derived up to order $e_{l-1}$.

Theorem 2. For $P$ in a Zariski open set of $\gamma$, the vector of $q$-Gaps at $P$ is $(1,2, \ldots, l)$.

Proof. The claim holds if we show that $E_{l}(P)=\{0\}$ for $P$ in the complement of a proper analytic subset of $\gamma^{*}$. Now, the space $E_{l}(P)$ is reduced to $\{0\}$ if and only if the following linear conditions are independent:

$$
\begin{cases}c_{1} g_{1}(0)+\ldots+c_{l} g_{l}(0) & =0 \\ c_{1} g_{1}^{\prime}(0)+\ldots+c_{l} g_{l}^{\prime}(0) & =0 \\ \vdots & \\ c_{1} g_{1}^{(l-1)}(0)+\ldots+c_{l} g_{l}^{(l-1)}(0) & =0\end{cases}
$$

The determinant of this system is a wronskian determinant that we will denote by $W_{0}(g)$. It then suffices to notice that the holomorphic function $z \mapsto W_{z}(g)$ cannot vanish identically in an open set of $\gamma^{*}$, as $g_{1}, \ldots, g_{l}$ are independent (see [2]). This proves Theorem 2 .

According to Remark 2, we have $M(\gamma, q, P)=e_{l}-1$, where $e_{l}$ is the last $q$-Gap at $P$. Generically on $\gamma, e_{l}$ is equal to $l$. Now, $l$ is $\left(\begin{array}{c}q+2 \\ 2\end{array}\right)$ when $q<p$, and $\left(\begin{array}{c}q+2 \\ 2\end{array}\right)-\left(\begin{array}{c}q-p+2 \\ 2\end{array}\right)$ when $q \geq p$. This completes the proof of Theorem 1.

Remark 4. Suppose $\gamma$ is not a line; take $q=1$. The vanishing locus of the corresponding wronskian describes the locus of $\gamma^{*}$ where the tangent line has contact at least 3 with $\gamma$, that is, the set of inflection points of $\gamma$.

Remark 5. Suppose the equation of $\gamma$ is nonreduced, that is, $F=f^{s}$, with $s \cdot \operatorname{deg} f=$ $p$. Then $M(F=0, q, P)=s \cdot M(f=0, q, P)$, and for sufficiently large $q$ and generic $P$ on $\gamma, M(F=0, q, P)=q p-\frac{p^{2}-3 p s+2 s^{2}}{2 s}$ is an increasing function of $s \in[1, \ldots, p]$. Then Bezout's bound $p q$ is reached for $s=p$, that is, when $\gamma$ is a multiple line.

Proposition 2. The function $P \mapsto M(\gamma, q, P)$ is upper semi-continuous on $\gamma^{*}$.

Proof. We need to see that for all $\sigma \in \mathbf{N}$, the set

$$
A_{\sigma}=\left\{P \in \gamma^{*}: M(\gamma, q, P)>\sigma\right\}
$$

is an analytic subset of $\gamma^{*}$. 
It is obvious that for all $P \in \gamma^{*}: M(\gamma, q, P) \geq l-1$. Thus, if $\sigma<l-1$, the set $A_{\sigma}$ is the whole of $\gamma^{*}$. If $\sigma=l-1, A_{\sigma}$ is the vanishing locus of the wronskian (2). In the case $\sigma>l-1$, let us denote by $L_{r}(z), r \geq 1$, the row vector $\left(g_{1}^{(r)}(z), \ldots, g_{l}^{(r)}(z)\right)$, and by $A_{0 i_{2} \ldots i_{l}}(z), 1 \leq i_{2}<i_{3}<\ldots<i_{l} \leq \sigma$, the $(l \times l)$ matrix whose rows are $L_{0}(z), L_{i_{2}}(z), \ldots, L_{i_{l}}(z)$. Then $A_{\sigma}$ is the intersection of the vanishing loci of $\operatorname{det} A_{0 i_{2} \ldots i_{l}}(z)$, over all possible indices $i_{2}, i_{3}, \ldots, i_{l}$. This is clearly an analytic subset of $\gamma^{*}$.

Example. Let $\gamma$ be the cubic curve $y-x^{3}=0$, let us take $q=2$ and $P\left(\alpha, \beta=\alpha^{3}\right)$ a point of $\gamma$. Since $q<\operatorname{deg} \gamma$, the vector space $E$ obtained by restricting $\mathbf{C}[x, y]_{2}$ on $\gamma$ has dimension $l=6$. The curve $\gamma$ is parametrized around $P$ by $\psi: t \mapsto$ $\left(t, 3 \alpha t^{2}+t^{3}\right)$. Let $Q=c_{1}+c_{2} x+\ldots+c_{6} y^{2}$ be a polynomial of $\mathbf{C}[x, y]_{2}$. Then $Q \circ \psi(t)$ belongs to $E_{6}(P)$ if and only if $\left(c_{1}, c_{2}, \ldots, c_{6}\right)$ satifies the linear system

$$
\begin{cases}c_{1} & =0, \\ c_{2} & =0, \\ 3 \alpha c_{3}+c_{4} & =0, \\ c_{3}+3 \alpha c_{5} & =0 \\ c_{5}+9 \alpha^{2} c_{6} & =0 \\ \alpha c_{6} & =0 .\end{cases}
$$

If $\alpha \neq 0$, the determinant of (3) is nonzero. Hence the vector of 2-Gaps at $P$ is $(1,2,3,4,5,6)$ and the only polynomial $Q, \operatorname{deg} Q \leq 2$, realizing contact 5 at $P$ (the osculating conic) is $-5 \alpha^{6}+24 \alpha^{5} x+40 \alpha^{3} y-45 \alpha^{4} x^{2}-15 \alpha^{2} x y+y^{2}$.

If $\alpha=0$, the vector of 2 -Gaps at the origin is $(1,2,3,4,5,7)$. The spaces $E_{5}(0)$ and $E_{6}(0)$ coincide; they are generated over $\mathbf{C}$ by $Q=y^{2}$. The maximal contact at the origin is 6 .

\section{ACKNOWLEDGMENT}

The author is grateful to C. Roche, J. M. Aroca, P. Fortuny and M. Chardin for fruitful discussions.

\section{REFERENCES}

[1] A. Gabrielov, Multiplicities of zeroes of polynomials on trajectories of polynomial vector fields and bounds on degree of nonholonomy, Math. Res. Lett. 2 (1995), 437-451. MR 97c:14055

[2] A. R. Magid, Lectures on differential Galois theory, University Lecture Series, volume 7, Amer. Math. Soc., Providence, RI, 1994. MR 95j:12008

[3] R. Miranda, Algebraic curves and Riemann surfaces, Graduate Studies in Mathematics, volume 5, chapter 7, Amer. Math, Soc., Providence, RI, 1995. MR 96f:14029

[4] Y. Yomdin, Oscillation of analytic curves, Proc. Amer. Math. Soc. 126, volume 2 (1998), 357-364. MR 98d:32033

Laboratoire de Mathématiques E. Picard, Université Paul Sabatier, UfR Mig, 118 route de Narbonne, 31062 Toulouse Cedex 4, France - and - Departmento de Álgebra, Geometría y Topología, Facultad de Ciencias, 47005 Valladolid, Spain

E-mail address: moura@picard.ups-tlse.fr 